Ferrata Storti Foundation

\title{
Venetoclax combines synergistically with FLT3 inhibition to effectively target leukemic cells in FLT3-ITD+ acute myeloid leukemia models
}

Haematologica 2021

Volume 106(4):1034-1046

\section{Correspondence: \\ ELISABETH A. LASATER \\ lasater.elisabeth@gene.com \\ DEEPAK SAMPATH \\ dsampath@ultragenyx.com}

Received: December 5, 2019.

Accepted: May 14, 2020.

Pre-published: May 15, 2020.

https://doi.org/10.3324/haematol.2019.244020

(C)2021 Ferrata Storti Foundation

Material published in Haematologica is covered by copyright. All rights are reserved to the Ferrata Storti Foundation. Use of published material is allowed under the following terms and conditions:

https://creativecommons.org/licenses/by-nc/4.0/legalcode. Copies of published material are allowed for personal or internal use. Sharing published material for non-commercial purposes is subject to the following conditions:

https://creativecommons.org/licenses/by-nc/4.0/legalcode, sect. 3. Reproducing and sharing published material for commercial purposes is not allowed without permission in writing from the publisher.
Raghuveer Singh Mali, ${ }^{1}$ Qi Zhang, ${ }^{2}$ Rosa Anna DeFilippis, ${ }^{3}$ Antonio Cavazos, ${ }^{2}$ Vinitha Mary Kuruvilla, ${ }^{2}$ Jayant Raman, ${ }^{3}$ Vidhi Mody, ${ }^{4}$ Edna F. Choo, ${ }^{4}$ Monique Dail, ${ }^{5}$ Neil P. Shah, ${ }^{3,6}$ Marina Konopleva, ${ }^{2}$ Deepak Sampath ${ }^{1 \% \#}$ and Elisabeth A. Lasater ${ }^{1 \#}$

${ }^{1}$ Department of Translational Oncology, Genentech, Inc., South San Francisco, CA; ${ }^{2}$ Department of Leukemia, The University of Texas M.D. Anderson Cancer Center, Houston, TX; ${ }^{3}$ Department of Medicine, Division of Hematology and Oncology, University of California, San Francisco, CA; ${ }^{4}$ Department of Drug Metabolism and Pharmacokinetics, Genentech, Inc., South San Francisco, CA; ${ }^{5}$ Oncology Biomarker Development, Genentech, Inc., South San Francisco, CA and ${ }^{6}$ Helen Diller Comprehensive Cancer Center, University of California, San Francisco, CA, USA

${ }^{\#} D S$ and EAL contributed equally as co-senior authors.

${ }^{\circ}$ Current address: Ultragenyx Pharmaceutical, Novato, CA, USA.

\section{ABSTRACT}

IT3 internal tandem duplication (FLT3-ITD) mutations account for -approximately $25 \%$ of adult acute myeloid leukemia (AML) cases and 1 are associated with poor prognosis. Venetoclax, a selective BCL-2 inhibitor, has limited monotherapy activity in relapsed/refractory AML with no responses observed in a small subset of FLT3-ITD+ patients. Further, FLT3-ITD mutations emerged at relapse following venetoclax monotherapy and combination therapy suggesting a potential mechanism of resistance. Therefore, we investigated the convergence of FLT3-ITD signaling on the BCL-2 family proteins and determined combination activity of venetoclax and FLT3-ITD inhibition in preclinical models. In vivo, venetoclax combined with quizartinib, a potent FLT3 inhibitor, showed greater anti-tumor efficacy and prolonged survival compared to monotherapies. In a patient-derived FLT3-ITD+ xenograft model, cotreatment with venetoclax and quizartinib at clinically relevant doses had greater anti-tumor activity in the tumor microenvironment compared to quizartinib or venetoclax alone. Use of selective BCL-2 family inhibitors further identified a role for BCL-2, BCL-X and MCL-1 in mediating survival in FLT3-ITD+ cells in vivo and highlighted the need to target all three proteins for greatest anti-tumor activity. Assessment of these combinations in vitro revealed synergistic combination activity for quizartinib and venetoclax but not for quizartinib combined with BCL- $X_{L}$ or MCL-1 inhibition. FLT3-ITD inhibition was shown to indirectly target both BCL- $X_{\mathrm{L}}$ and MCL-1 through modulation of protein expression, thereby priming cells toward BCL-2 dependence for survival. These data demonstrate that FLT3-ITD inhibition combined with venetoclax has impressive anti-tumor activity in FLT3-ITD+ AML preclinical models and provides strong mechanistic rational for clinical studies.

\section{Introduction}

Fms-like tyrosine kinase 3 (FLT3) is one of the most commonly mutated genes in adult acute myeloid leukemia (AML) with internal tandem duplication (FLT3-ITD) mutations identifiable in approximately $25 \%$ of cases ${ }^{1}$ and activating tyrosine kinase domain (FLT3-TKD) mutations occurring in about $7 \%$ of cases. ${ }^{2}$ Both FLT3ITD and FLT3-TKD are ligand-independent, constitutively activated kinases that signal through the canonical downstream pathways phosphoinositide 3-kinase $(\mathrm{PI} 3 \mathrm{~K})$ and mitogen-activated protein kinases (RAS-MAPK) ${ }^{3}$ and broadly impact cell 
A

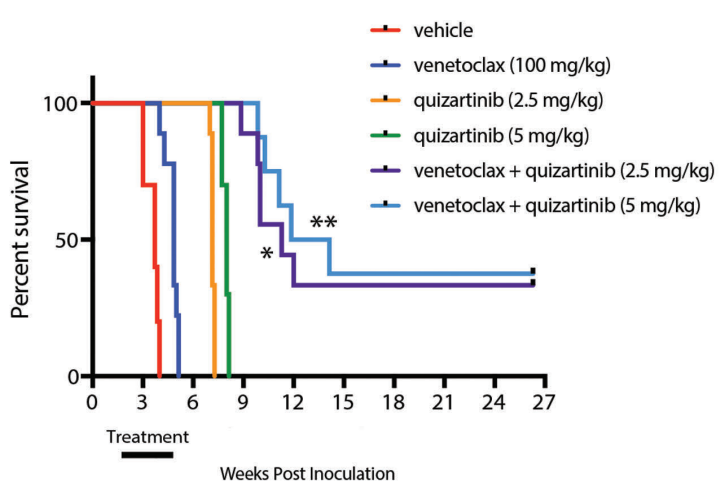

C venetoclax (100 mg/kg) $\quad$ venetoclax + quizartinib $(2.5 \mathrm{mg} / \mathrm{kg})$

$\triangle$ quizartinib $(2.5 \mathrm{mg} / \mathrm{kg})$ venetoclax + quizartinib $(5 \mathrm{mg} / \mathrm{kg})$

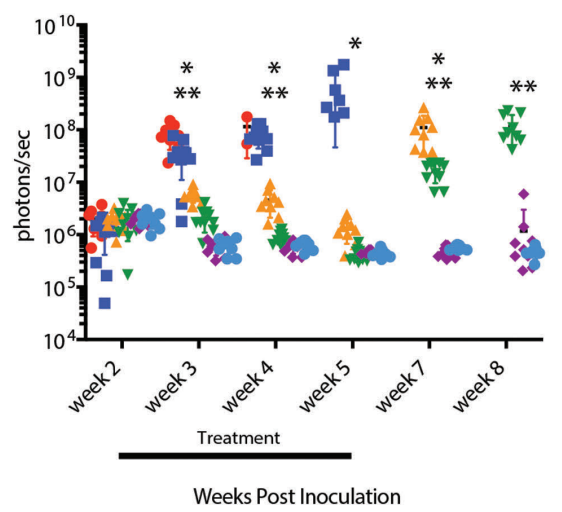

B

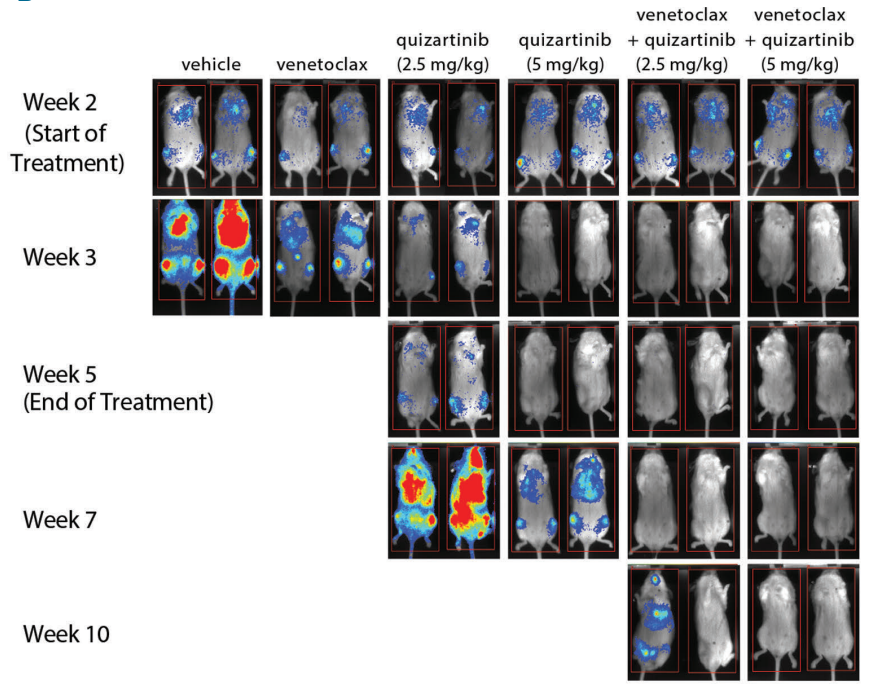

D

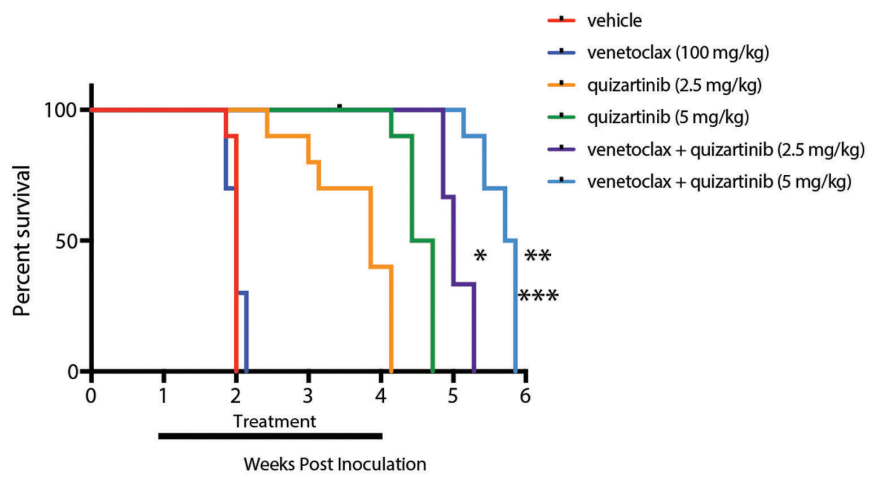

E

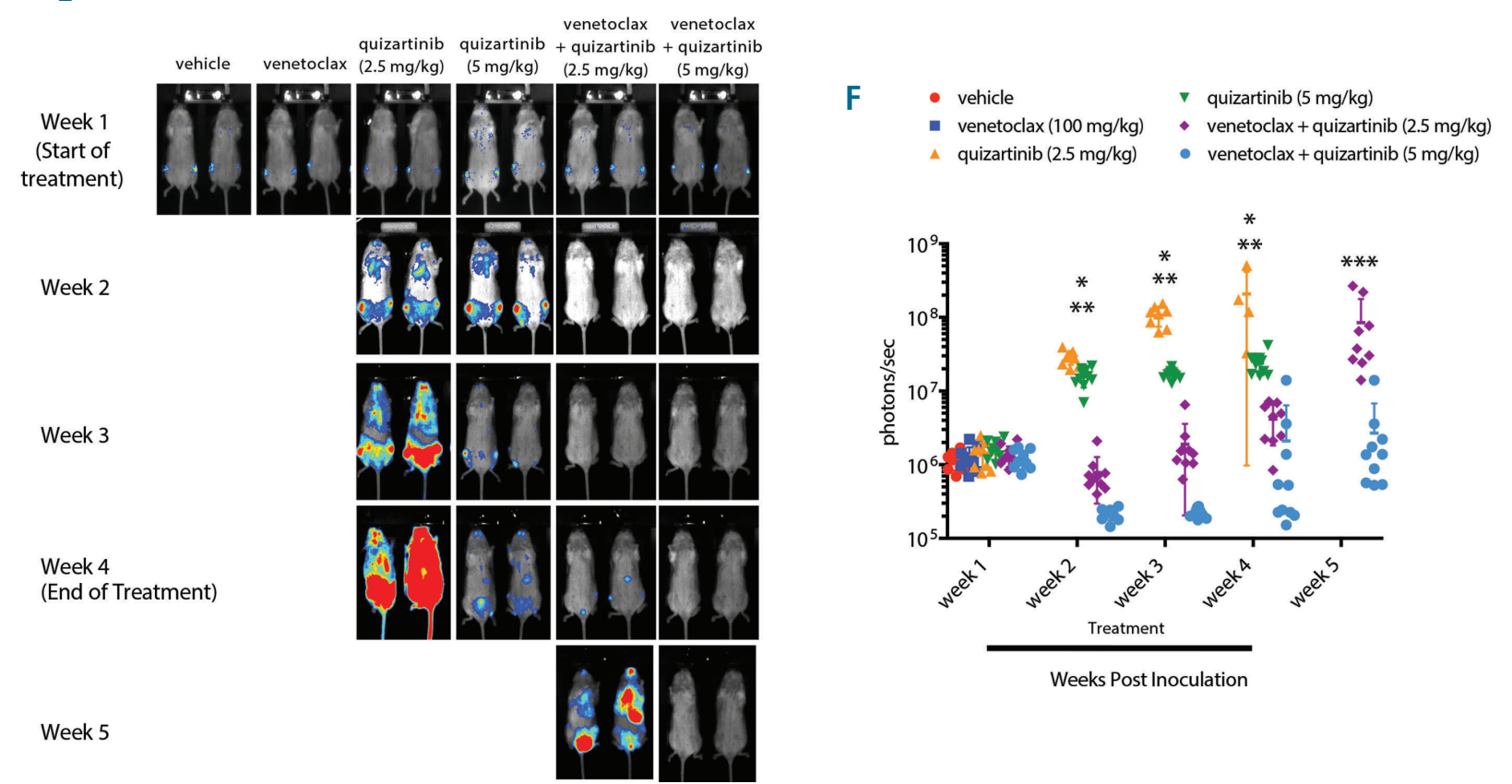


Figure 1 (previous page). Venetoclax combined with quizartinib prolongs survival and reduces tumor burden in FLT3-ITD+ xenograft models. NOD/SCID/IL-2R $\gamma^{\text {null }}$ (NSG) mice were engrafted with luciferase expressing MV4;11 or Molm13 cells and leukemic cell engraftment was confirmed by bioluminescence imaging (BLI) and treatment began 14 days post-inoculation for the MV4;11 model and 7 days post-inoculation in the Molm13 model. Mice were treated orally with 100 mg/kg venetoclax, $2.5 \mathrm{mg} / \mathrm{kg}$ or $5 \mathrm{mg} / \mathrm{kg}$ quizartinib, or the combination once daily for 21 continuous days and overall survival and disease burden was assessed. (A) KaplanMeier survival curve for MV4;11-engrafted mice. $\mathrm{N}=8-10$ animals/group and median survival and statistical significance were determined by log-rank test $* P<0.0001$ for venetoclax $+2.5 \mathrm{mg} / \mathrm{kg}$ quizartinib vs. $2.5 \mathrm{mg} / \mathrm{kg}$ quizartinib; $* * P<0.0001$ for venetoclax $+5 \mathrm{mg} / \mathrm{kg}$ quizartinib vs. $5 \mathrm{mg} / \mathrm{kg}$ quizartinib. (B) Representative BLI for MV4;11-engrafted mice for each group at indicated time point. (C) Quantitation of the BLI signal from MV4;11-engrafted mice in each group at indicated time post-inoculation: $* P<0.0001, P=0.0002, P=0.001$ and $P=0.0004$ for venetoclax $+2.5 \mathrm{mg} / \mathrm{kg}$ quizartinib vs. $2.5 \mathrm{mg} / \mathrm{kg}$ quizartinib for week 3,4 , 5 and 7 , respectively, by unpaired t-test for each time point; $* * P=0.0004, P=0.0247, P<0.0001$ and $P=0.0001$ for venetoclax $+5 \mathrm{mg} / \mathrm{kg}$ quizartinib vs. $5 \mathrm{mg} / \mathrm{kg}$ quizartinib for week $3,4,7$ and 8 , respectively, by unpaired t-test for each time point. (D) Kaplan-Meier curve for Molm13-engrafted mice. $\mathrm{N}=10$ animals/group and survival and statistical significance were determined by log-rank test: $* P<0.0001$ for venetoclax $+2.5 \mathrm{mg} / \mathrm{kg}$ quizartinib vs. $2.5 \mathrm{mg} / \mathrm{kg}$ quizartinib; $* * P<0.0001 \mathrm{for}$ venetoclax $+5 \mathrm{mg} / \mathrm{kg}$ quizartinib vs. $5 \mathrm{mg} / \mathrm{kg}$ quizartinib; and $* * * P<0.0001$ for venetoclax $+5 \mathrm{mg} / \mathrm{kg}$ quizartinib vs. venetoclax $+2.5 \mathrm{mg} / \mathrm{kg}$ quizartinib. (E) Representative BLI for Molm13-engrafted mice at indicated time point. (F) Quantitation of BLI signal from Molm13-engrafted mice at indicated time point post-inoculation: $* P<0.0001, P<0.0001$ and $P=0.0086$ for venetoclax $+2.5 \mathrm{mg} / \mathrm{kg}$ quizartinib vs. $2.5 \mathrm{mg} / \mathrm{kg}$ quizartinib for week 2 , 3 and 4 , respectively, by unpaired t test for each timepoint; $* * P<0.0001$ for venetoclax $+5 \mathrm{mg} / \mathrm{kg}$ quizartinib vs. $5 \mathrm{mg} / \mathrm{kg}$ quizartinib for week 2 , 3 and 4 by unpaired t-test for each timepoint; and $* * * P=0.0129$ for venetoclax $+5 \mathrm{mg} / \mathrm{kg}$ quizartinib vs. venetoclax $+2.5 \mathrm{mg} / \mathrm{kg}$ quizartinib at week 5 by unpaired t-test.

proliferation, differentiation, and survival. Notably, FLT3ITD also activates STAT5, a distinguishing feature from FLT3-TKD and ligand-stimulated wild-type (WT) FLT3., ${ }^{4}$

Intrinsic apoptosis regulates survival through balancing anti- and pro-apoptotic proteins. The BCL-2 family of anti-apoptotic proteins includes B-cell lymphoma 2 (BCL2), B-cell lymphoma-extra-large (BCL- $\mathrm{X}_{\mathrm{L}}$ ) and myeloid cell leukemia 1 (MCL-1) that bind and neutralize pro-apoptotic BCL-2 homology 3 (BH3)-only proteins and pro-apoptotic effector proteins, BCL-2-associated X protein (BAX) and BCL-2 antagonist/killer (BAK), ${ }^{6}$ preventing induction of apoptosis. The development of $\mathrm{BH} 3$ mimetic compounds, including venetoclax (ABT-199/GDC-0199), that specifically target individual BCL-2 family members has helped clarify dependence of cancer cells on BCL-2,7 BCL$\mathrm{X}_{\mathrm{L}}^{8,9}$ and/or MCL- $1^{10,11}$ and also revealed mechanisms of venetoclax resistance, including upregulation of BCL- $\mathrm{X}_{\mathrm{L}}$ and/or MCL-1. ${ }^{12}$

Venetoclax, a highly potent, specific inhibitor of BCL-2, has preclinical and clinical activity across a range of hematologic malignancies. ${ }^{13-17}$ In AML, while venetoclax has limited monotherapy activity ${ }^{14}$ venetoclax combined with low-dose cytarabine (LDAC) or hypomethylating agents (HMA) has broad activity across mutation subsets. ${ }^{18,19}$ In the venetoclax monotherapy trial, ${ }^{14}$ new FLT3-ITD mutations emerged at progression in 4 of 15 patients with initial venetoclax response and 3 of 3 patients with baseline FLT3-ITD mutations showed no measurable reduction in bone marrow (BM) blasts. ${ }^{20}$ Initial molecular analysis of samples from the venetoclax combination trials revealed that relapse may also be associated with FLT3-ITD mutations. ${ }^{21}$ In 25 patients evaluated at relapse, FLT3-ITD mutations expanded in three patients treated with venetoclax plus HMA and new FLT3-ITD mutations were detected in two patients treated with venetoclax plus LDAC. While patient numbers reported in these studies are low, the association of FLT3-ITD mutations with primary resistance and emergence of FLT3-ITD mutations at relapse suggests that mutant FLT3 may influence sensitivity to venetoclax and warrants further investigation.

Resistance to apoptosis can be achieved through oncogenic signaling or transcriptional regulation that alter expression of apoptotic proteins. RAS-MAPK, PI3K and the Janus kinase (JAK)-STAT pathways can all regulate anti- and pro-apoptotic proteins. ${ }^{22-24}$ As such, STAT5, a known transcriptional regulator of BCL- $\mathrm{X}_{\mathrm{L}}$ and $\mathrm{BCL}-2,{ }^{25}$ can also regulate MCL-1 expression in FLT3-ITD+ cells. ${ }^{26}$ Given that FLT3-ITD regulates multiple survival pathways and is linked to increased BCL- $\mathrm{X}_{\mathrm{L}}^{27-30}$ and MCL-1 ${ }^{26,29}$ expression, which can promote venetoclax resistance, there is strong scientific rationale to investigate $\mathrm{BH} 3$ mimetics combined with FLT3-ITD inhibition. Ma et al. described combination activity of venetoclax and the FLT3 inhibitors midostaurin and gilteritinib in FLT3-ITD+ AML models. ${ }^{31}$ However, direct comparison of BCL-2, BCL- $\mathrm{X}_{\mathrm{L}}$ and MCL-1 inhibition in reducing survival when combined with FLT3-ITD inhibition has not been investigated, nor has the role of individual signaling pathways downstream of FLT3-ITD in regulating BCL-2 family members. Therefore, we aimed to thoroughly investigate the combined efficacy of FLT3-ITD and BCL2 family inhibition using potent and selective pharmacologic inhibitors in pre-clinical models of FLT3-ITD+ AML. We describe herein the superiority of targeting BCL-2, rather than BCL-X $\mathrm{X}_{\mathrm{L}}$ or MCL-1, in combination with FLT3 inhibition in FLT3-ITD+ AML. Given that venetoclax is presently being tested in AML clinical trials, our preclinical data provides a strong mechanistic rational for further evaluation of venetoclax combinations with FLT3-ITD inhibitors for the treatment of FLT3-ITD+ patients.

\section{Methods}

\section{Orthotopic cell line xenograft models}

Cell line xenograft studies were approved by Genentech's Institutional Animal Care and Use Committee (IACUC) and adhere to the Eighth Edition of the Guide for the Care and Use of Laboratory Animals (NRC 2011). NOD/SCID/IL-2R $\gamma^{\text {null }}$ (NSG) mice (Jackson Laboratory, Bar Harbor, ME) were housed in autoclaved individually ventilated cages. Eight to 10 week old mice were pre-conditioned with busulfan ( $20 \mathrm{mg} / \mathrm{kg}$; Sigma) by intraperitoneal administration 24 hours prior to cell line inoculation. Luciferase-positive AML cell lines $\left(2 \times 10^{6}\right.$ cells) were suspended in Hanks' balanced salt solution (HBSS) and injected via tail vein. Engraftment of leukemic cells and disease burden was determined by bioluminescence imaging (BLI). Equally engrafted mice were grouped out based on BLI at 7 (Molm13) or 14 days (MV4;11) post inoculation and treated as described for 21 continuous days. Animals were monitored for signs of disease progression and euthanized at first measurement of greater than $20 \%$ weight loss or when reaching any humane endpoint.

\section{Primary patient-derived xenograft models}

Primary patient samples were collected and utilized at the M.D. Anderson Cancer Center (MDACC). All patients gave informed consent in accord with the Declaration of Helsinki under Institutional Review Board-approved protocols. Studies were approved by the MDACC IACUC and adhere to the Eighth Edition of the Guide for the Care and Use of Laboratory Animals (NRC 2011). Seven week old NSG mice were pre-conditioned by 
A

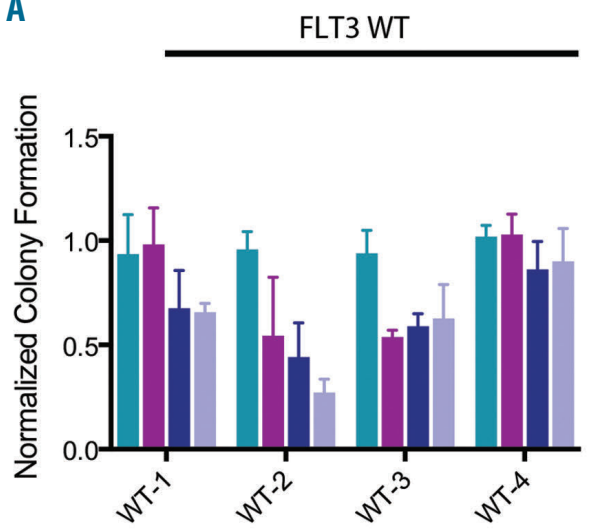

B

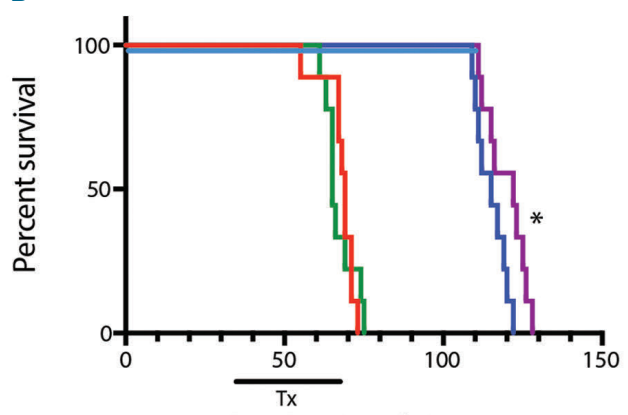

Days Post Inoculation

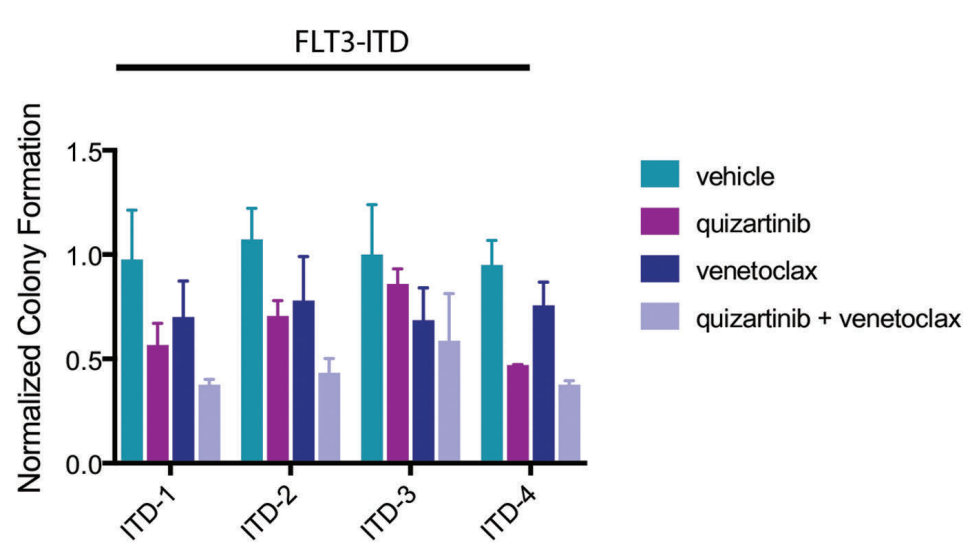

FLT3-ITD

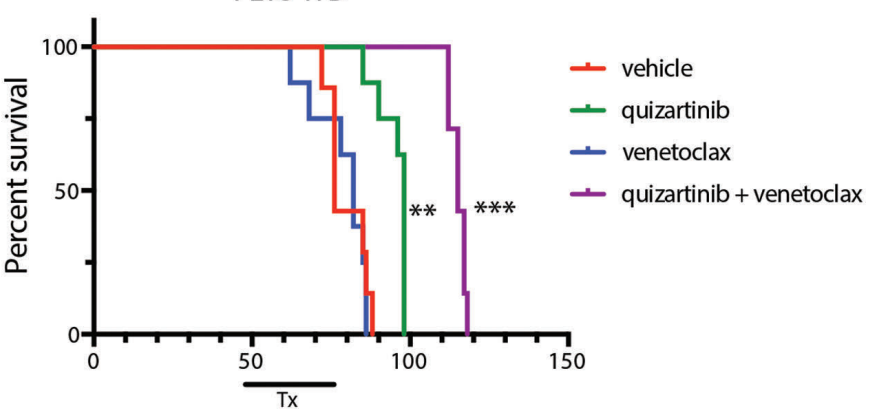

Days Post Inoculation

Figure 2. Venetoclax combined with quizartinib has greater anti-tumor efficacy against primary FLT3-ITD+ acute myeloid leukemia models. (A) Primary patient samples were treated with $20 \mathrm{nM}$ quizartinib, $50 \mathrm{nM}$ venetoclax or the combination in methylcellulose in triplicate for 14 days and colony forming units were counted. Data were normalized to the median colony number for the vehicle-treated cells for each sample and plotted as mean + standard deviation. (B) NOD/SCID/IL-2R $\gamma^{\text {null }}$ (NSG) mice were engrafted with primary samples from Fms-like tyrosine kinase 3 wild-type (FLT3-WT) or FLT3 internal tandem duplication (FLT3-ITD+) patients and treated orally with $5 \mathrm{mg} / \mathrm{kg}$ quizartinib, $100 \mathrm{mg} / \mathrm{kg}$ venetoclax or the combination once daily for 28 days and efficacy was assessed. Kaplan-Meier survival curve for FLT3-WT and FLT3-ITD+ models. N=7-9 animals/group and median survival and statistical significance were determined by log-rank test: * $P<0.0001$ for venetoclax vs. vehicle or quizartinib in FLT3-WT model. $* * P=0.0004$ for quizartinib vs. vehicle or venetoclax and $* * * P=0.0003$ for quizartinib + venetoclax vs. quizartinib in the FLT3-ITD+ model.

sublethal irradiation (250 cGY) 24 hours prior to cell inoculation. FLT3 WT or FLT3-ITD+ primary patient samples ( $1 \times 10^{6}$ cells) were suspended in Phosphate-buffered saline (PBS) and inoculated through tail vein injection. Engraftment and disease burden were determined by co-staining for human and murine anti-CD45 (BioLegend, San Diego, CA) in peripheral blood samples. Mice were grouped out based upon engraftment (WT average 2.2\% engraftment; ITD average $0.8 \%$ engraftment) and treated as described for 28 continuous days. Animals were monitored for signs of disease progression and euthanized at first measurement of greater than $20 \%$ weight loss or when reaching any humane endpoint.

\section{BLISS analysis}

Details are provided in the Online Supplementary Appendix.

\section{BH3 profiling}

Details are provided in the Online Supplementary Appendix.

\section{Statistical analysis}

Statistical comparisons included unpaired t-test or one-way ANOVA with Tukey post hoc test or Dunnett post-test, as indicated with $P<0.05$ considered statistically significant. Kaplan-Meier survival analysis was used for in vivo studies and significance was determined by log-rank test with $P<0.05$ considered significant.
All analyses were completed using Prism software package (version 7; Graphpad Software, La Jolla, CA, USA).

\section{Results}

\section{Venetoclax combined with FLT3-ITD inhibition} prolonged survival of FLT3-ITD+ Ieukemic mice in vivo

In order to explore the impact FLT3-ITD on venetoclax activity, we assessed venetoclax treatment in combination with the selective FLT3 inhibitor quizartinib (AC220) ${ }^{32}$ in FLT3-ITD+ cell line xenograft models (MV4;11 and Molm13). Mice with established leukemia were treated orally with vehicle, venetoclax $(100 \mathrm{mg} / \mathrm{kg})$, quizartinib (2.5 or $5 \mathrm{mg} / \mathrm{kg}$ ), or the combination for 21 continuous days. Consistent with clinical data, venetoclax alone demonstrated little to no efficacy based on survival with marginal improvement in the MV4;11 model (Figure 1AC) and no anti-tumor activity in the Molm13 model (Figure 1D-F), while quizartinib significantly reduced disease burden and increased survival compared to vehicle in both models (Figure 1A-F). Venetoclax combined with quizartinib led to further improvement in survival and reduction in tumor burden compared to quizartinib alone (Figure 1A-F). Additionally, in the MV4;11 model a subset 
A
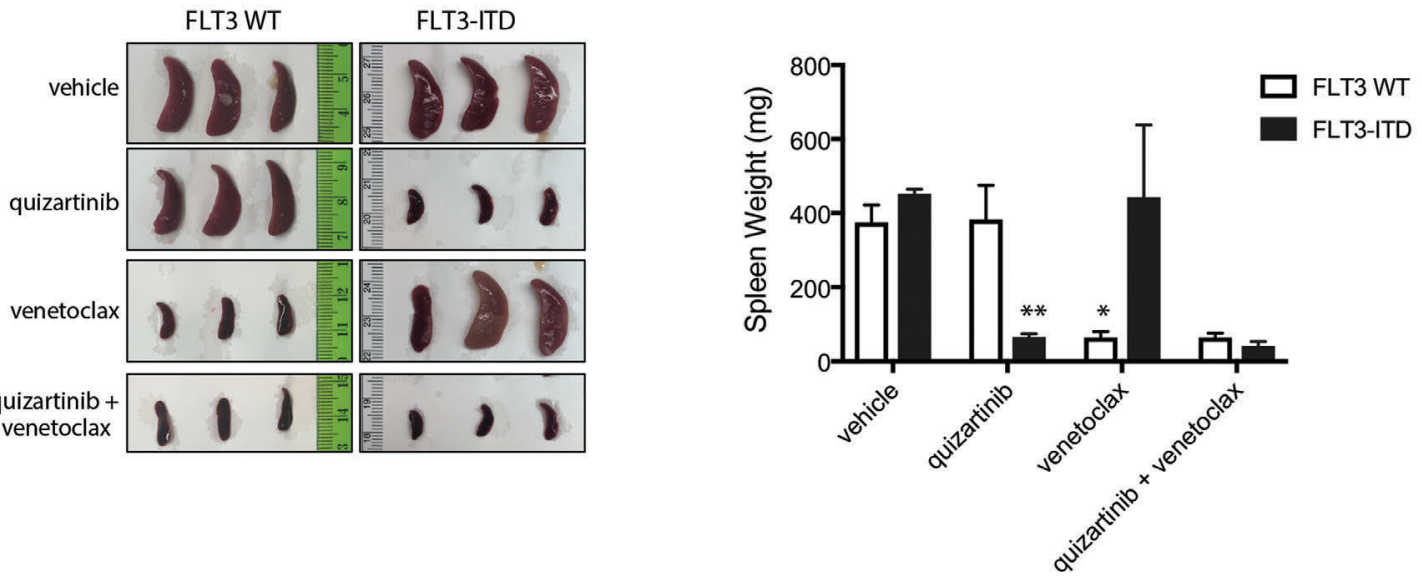

B Peripheral Blood
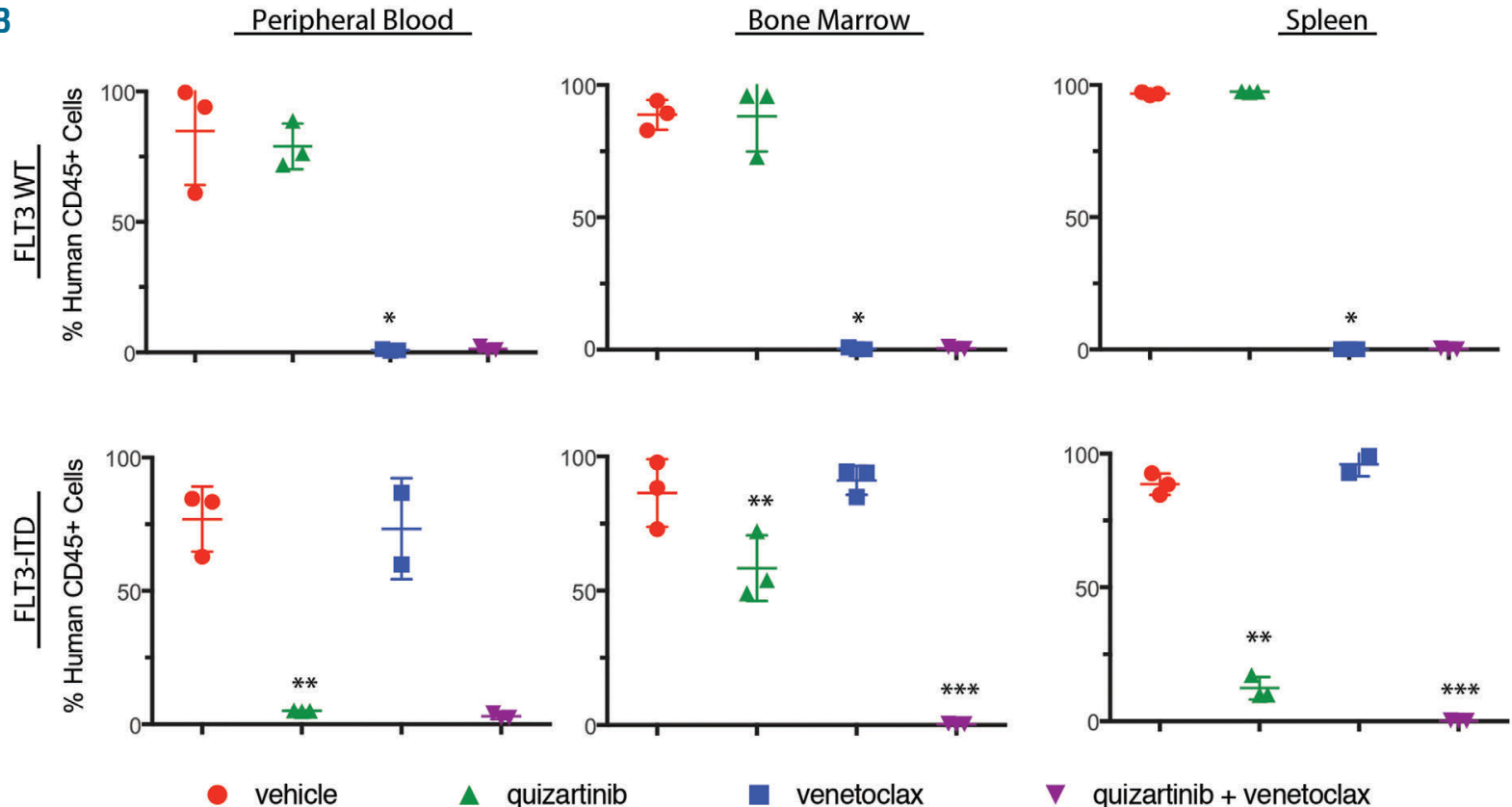

$\nabla$ quizartinib + venetoclax

Figure 3. Venetoclax combined with quizartinib to reduce disease burden in the tumor microenvironment of patient-derived FLT3-ITD+ xenograft models. NOD/SCID/IL-2R $\gamma^{\text {null }}$ (NSG) mice were engrafted with primary samples from Fms-like tyrosine kinase 3 wild-type (FLT3-WT) or FLT3 internal tandem duplication (FLT3ITD+) patients and were treated orally with $5 \mathrm{mg} / \mathrm{kg}$ quizartinib, $100 \mathrm{mg} / \mathrm{kg}$ venetoclax or the combination once daily for 28 days. (A) Spleen images and spleen weight at the end of 28 days of dosing. Data is represented as average + standard deviation $(n=3)$. * $P=0.0004$ for venetoclax vs. quizartinib for FLT3-WT model and $* * P=0.0068$ for quizartinib vs. venetoclax for FLT3-ITD + model by one-way ANOVA with Tukey post hoc test. (B) Percentage of human CD45 ${ }^{+}$cells in peripheral blood, bone marrow and spleen at the end of dosing. Data is represented as average + standard deviation $(n=3)$. $* P<0.0001$ for venetoclax vs. quizartinib in the FLT3-WT model in the peripheral blood, bone marrow and spleen; $* * P=0.0005, P=0.01$ and $P<0.0001$ for quizartinib vs. venetoclax for peripheral blood, bone marrow and spleen, respectively, for FLT3-ITD+ model; and $* * * P=0.0002$ and $P=0.0159$ for quizartinib + venetoclax vs. quizartinib for bone marrow and spleen, respectively, for FLT3-ITD+ model by one-way ANOVA with Tukey post hoc test.

of mice remained disease-free showing long-term survival following combination treatment with venetoclax that was not observed with quizartinib monotherapy. All drug treatments were well tolerated based on minimal changes in body weight (Online Supplementary Figure S1). Importantly, combination activity was demonstrated at clinically relevant doses, as $5 \mathrm{mg} / \mathrm{kg}$ dose of quizartinib achieved Cmax of $\sim 1 \mu \mathrm{M}$ in NSG mice (Online Supplementary Figure S2), similar to the clinically efficacious dose of $60 \mathrm{mg} /$ day in patients. ${ }^{33}$

\section{FLT3-ITD inhibition combines with venetoclax in primary patient samples}

Studies were expanded to primary AML patient samples to confirm combination activity ex vivo. FLT3-WT and
FLT3-ITD+ primary samples were treated with quizartinib, venetoclax or the combination of both drugs and colony forming units were determined after 14 days. In 3 of 4 FLT3-ITD+ samples, venetoclax plus quizartinib impaired colony formation greater than each agent alone (Figure 2A), suggesting that the co-treatment targeted more FLT3-ITD+ progenitor cells, which is an important factor related to greater clinical response.

In vivo efficacy of quizartinib and venetoclax was also investigated in FLT3-WT and FLT3-ITD+ patient-derived xenograft (PDX) models (see Online Supplementary Figure $S 3 A$ for cytogenetic characteristics). Following engraftment, mice were treated orally with vehicle, venetoclax (100 $\mathrm{mg} / \mathrm{kg}$ ), quizartinib $(5 \mathrm{mg} / \mathrm{kg})$, or the combination for 28 continuous days. Quizartinib prolonged survival of the 
A

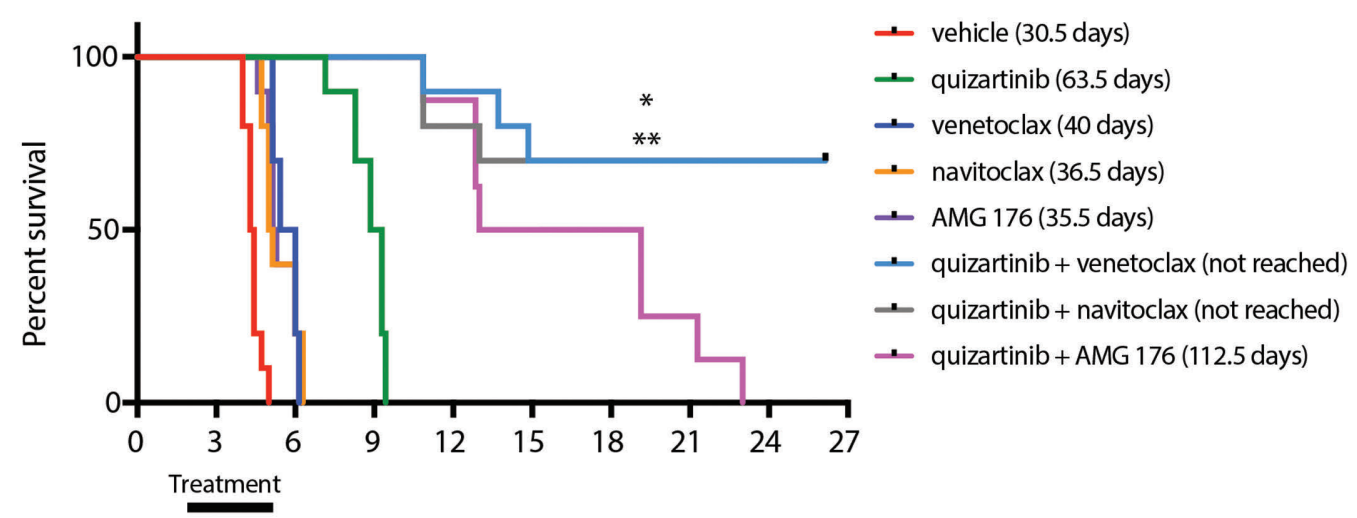

Weeks Post Inoculation

B

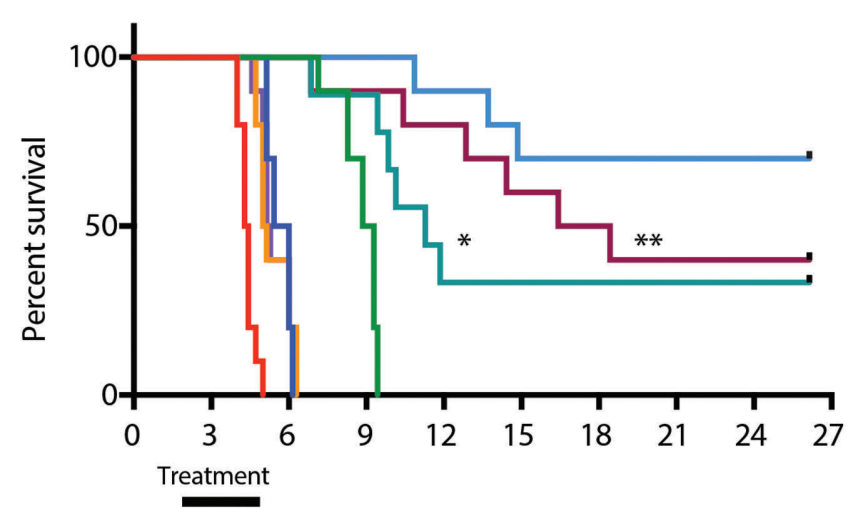

Weeks Post Inoculation

C

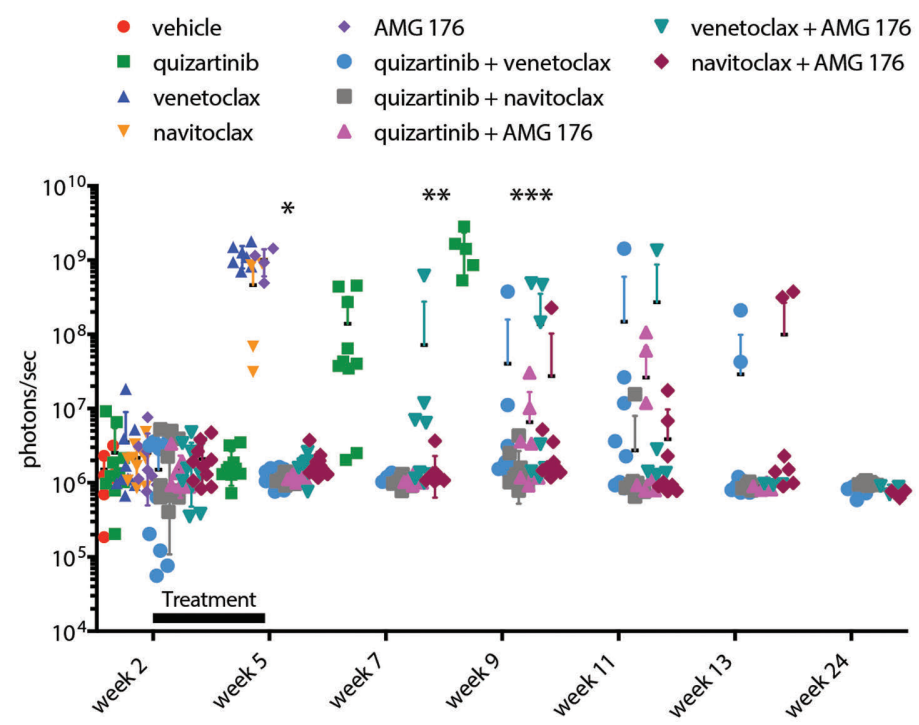

Weeks Post Inoculation
+ vehicle (30.5 days)

+ quizartinib (63.5 days)

土 venetoclax (40 days)

- navitoclax (36.5 days)

+ AMG 176 (35.5 days)

- venetoclax + AMG 176 (79 days)

ـ navitoclax + AMG 176 (122 days)

- quizartinib + venetoclax (not reached)

Figure 4. BCL-2, BCL- $X_{L}$ and MCL-1 contribute to survival of FLT3-ITD+ cells in vivo. NOD/SCID/IL-2R $\gamma^{\text {nul }}$ (NSG) mice were engrafted with luciferase expressing MV4;11 cells and leukemic engraftment was confirmed by BLI and treatment began 14 days post inoculation. Mice were treated orally with $5 \mathrm{mg} / \mathrm{kg}$ quizartinib, $100 \mathrm{mg} / \mathrm{kg}$ venetoclax, $100 \mathrm{mg} / \mathrm{kg}$ navitoclax or $10 \mathrm{mg} / \mathrm{kg}$ AMG 176 as single agents or in combinations as indicated once daily for 21 days. All treatment groups were run concurrently, however for clarity survival data has been split between panels $A$ and $B$ and vehicle, quizartinib, venetoclax, navitoclax and AMG 176 single agent groups have been plotted in both panels for reference. (A) Kaplan-Meier survival curves for quizartinib in combination with AMG 176 , venetoclax or navitoclax. $\mathrm{N}=8-10$ animals/group. Median survival and statistics were determined by log-rank test: $* P=0.0059$ for quizartinib + venetoclax vs. quizartinib + AMG 176; and $* * P=0.0095$ for quizartinib + navitoclax vs. quizartinib + AMG 176. (B) Kaplan-Meier survival curves for AMG 176 in combination with venetoclax or navitoclax. $\mathrm{N}=8-10$ animals/group. Median survival and statistics were determined by log-rank test: $* P=0.0008$ for venetoclax + AMG 176 vs. quizartinib; and $* * P=0.0002$ for navitoclax + AMG 176 vs. quizartinib. (C) Quantitation of the bioluminescence imaging (BLI) signal from MV4;11-engrafted mice in each group at indicated time post-inoculation: $* P<0.005$ for quizartinib single agent and all combinations vs. venetoclax, AMG 176 or navitoclax single agents at week 5 by one-way ANOVA with Tukey post-test; $* * P<0.05$ for quizartinib + venetoclax, quizartinib + navitoclax, quizartinib + AMG 176 and navitoclax + AMG 176 vs. quizartinib single agent on week 7 by one-way ANOVA with Dunnett post-test; $* * * P=0.0001$ for quizartinib + venetoclax, quizartinib + navitoclax, quizartinib + AMG 176, venetoclax + AMG 176 and navitoclax + AMG 176 vs. single agent quizartinib at week 9 by one-way ANOVA with Dunnett post-test. 
FLT3-ITD+ model whereas venetoclax prolonged survival of the FLT3-WT model (Figure 2B). Venetoclax combined with quizartinib further improved survival of the FLT3ITD+ model compared to quizartinib alone, while no combination effect was observed in the FLT3-WT model (Figure 2B). Analysis of leukemia cells from vehicle-treated mice showed that FLT3-WT cells had elevated BCL-2 protein expression relative to $\mathrm{BCL}-\mathrm{X}_{\mathrm{L}}$ or $\mathrm{MCL}-1$, which may account for venetoclax sensitivity. Conversely, the FLT3ITD+ cells showed elevated MCL-1 expression compared

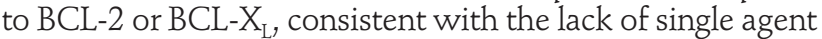
venetoclax activity (Online Supplementary Figure S3B).

A cohort of drug-treated mice ( $n=3$ /group) were assessed at the end of dosing. Venetoclax reduced splenomegaly and leukemic cells in peripheral blood (PB), BM and spleen (Figure 3A-B; Online Supplementary Figure $S 3 C$ ) in the FLT3-WT model. In the FLT3-ITD+ model, quizartinib treatment significantly reduced splenomegaly and leukemic blasts in the $\mathrm{PB}, \mathrm{BM}$ and spleen while venetoclax demonstrated little activity (Figure 3A-B; Online Supplementary Figure S3C). Although quizartinib monotherapy profoundly reduced FLT3-ITD+ PB blasts, significant blasts were still present in the BM $(\sim 50 \%)$ and spleen ( 12\%; Figure 3B; Online Supplementary Figure S3C). Notably, the addition of venetoclax to quizartinib reduced leukemic cells in the BM and spleen to less than $1 \%$ (Figure 3B; Online Supplementary Figure S3C). Treatment was well tolerated as determined by minimal changes in body weight (Online Supplementary Figure S3D). These results further demonstrate that venetoclax, when combined with quizartinib, is more efficacious than either single agent alone in FLT3-ITD+ primary AML localized within a biologically relevant tumor microenvironment at clinically achievable doses.

A

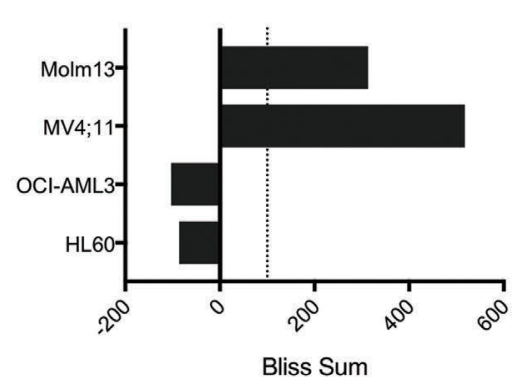

B
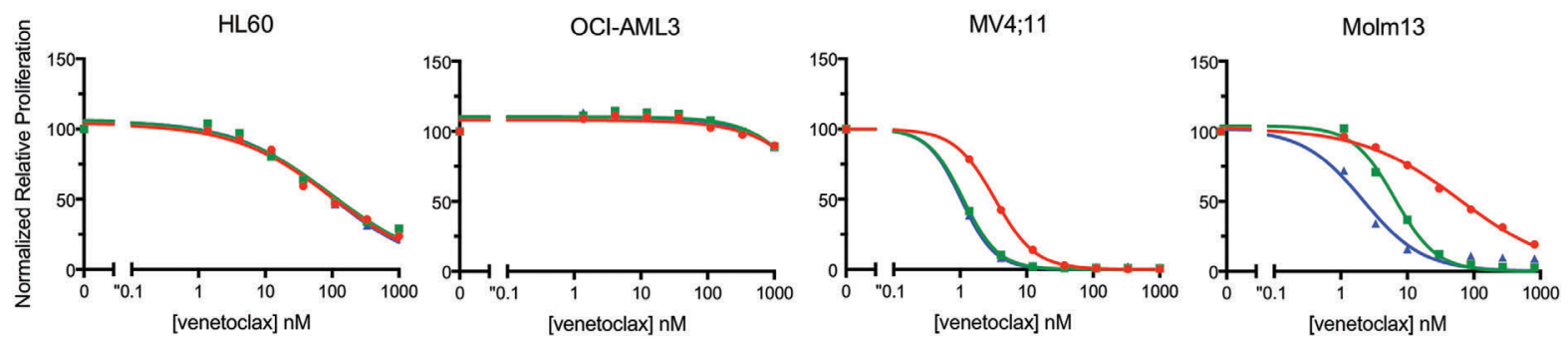

$\rightarrow$ quizartinib $(0 \mathrm{nM}) \quad \rightarrow$ quizartinib $(3.33 \mathrm{nM}) \quad \rightarrow$ quizartinib $(10 \mathrm{nM})$

C

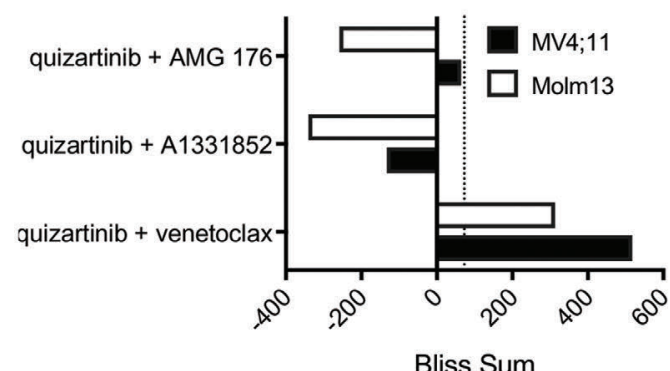

D

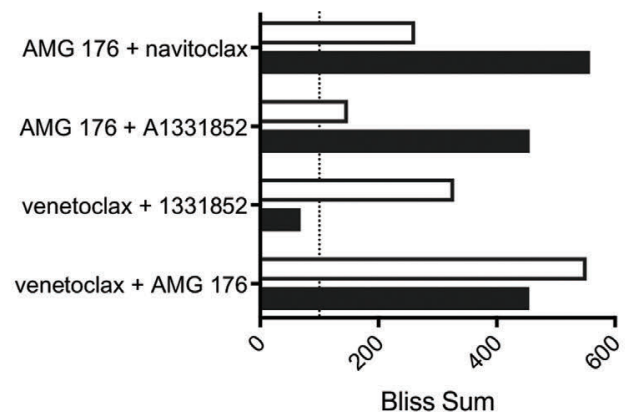

MV4;11

Molm13

Figure 5. Venetoclax synergistically combined with quizartinib in FLT3-ITD+ cell lines. (A) Cell lines were treated for 48 hours with venetoclax, quizartinib or the combination at indicated concentrations. ATP content was determined by CellTiter-Glo and Bliss sums were calculated and plotted for each cell line. Bliss sum of $>100$ is highly synergistic. (B) Cell lines were treated for 48 hours with quizartinib, venetoclax or the combination as indicated and cell viability was assessed by CellTiterGlo. Values are normalized to the average of the untreated samples for each cell line. (C) Fms-like tyrosine kinase 3 (FLT3) internal tandem duplication (FLT3-ITD+) cell lines were treated for 48 hours with combinations of quizartinib and venetoclax, AMG 176 or A1331852 as indicated. ATP content was determined by CellTiterGlo and Bliss sums were calculated and plotted for each cell line. (D) FLT3-ITD+ cell lines were treated for 48 hours with combinations of venetoclax, AMG 176, A1331852 or navitoclax as indicated. ATP content was determined by CellTiter-Glo and Bliss sums were calculated and plotted for each cell line. 
BCL-2, BCL- $X_{L}$ and MCL-1 promote survival of FLT3-ITD+ cells in vivo

In order to understand the role of BCL- $X_{L}$ and MCL-1 in FLT3-ITD mediated survival in vivo, we utilized selective inhibitors to pharmacologically assess the contributions of the anti-apoptotic proteins. Drug combinations of quizartinib, venetoclax, navitoclax (dual BCL-2/BCL-X inhibitor) $^{8}$ and AMG 176 (MCL-1 inhibitor) ${ }^{11}$ were tested in the MV4;11 model at well-tolerated and clinically rele- vant doses. AMG 176 dosed at $10 \mathrm{mg} / \mathrm{kg}$ achieved a $\mathrm{C}_{\max }$ of $\sim 2 \mu \mathrm{M}$ in peripheral blood of NSG mice (Online Supplementary Figure S2), which is within the cumulative weekly dose range recently reported ${ }^{11}$ and $100 \mathrm{mg} / \mathrm{kg}$ dose of navitoclax overlaps with clinically achievable exposure. ${ }^{8,9}$ Other than quizartinib, single agents targeting the anti-apoptotic proteins showed minimal improvement in survival compared to vehicle control while all combinations with quizartinib enhanced its single agent activity.
A

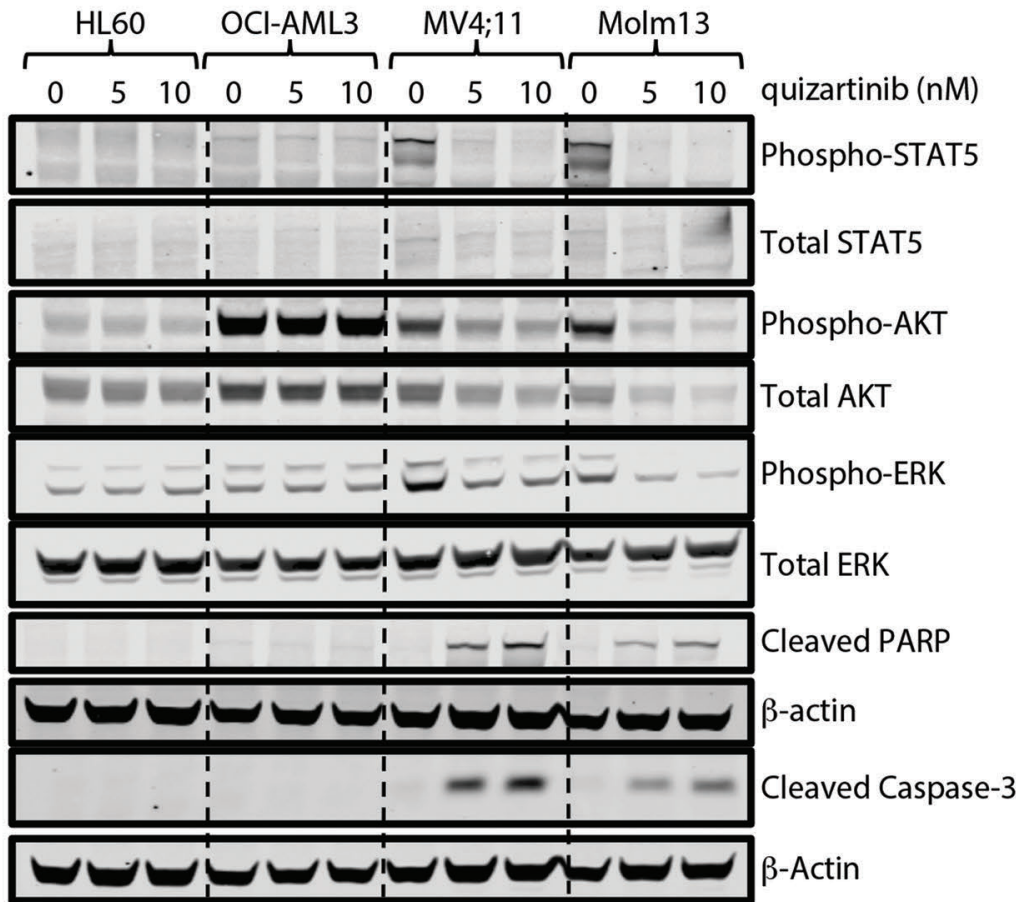

Figure 6. FLT3-ITD signaling regulates the expression of $\mathrm{BCL}-\mathrm{X}_{\mathrm{L}}$ and MCL-1 in vitro. Fms-like tyrosine kinase 3 wild-type (FLT3-WT) (HL60 and $\mathrm{OCl}-\mathrm{AML} 3$ ) and FLT3 interna tandem duplication (FLT3-ITD+) (MV4;11 and Molm13) cell lines treated for 24 hours with indicated concentration of quizartinib and cell lysates analyzed by western blot for (A) FLT3-ITD downstream effector proteins and (B) BCL-2 family proteins as indicated. (C) Cell lines were treated with $10 \mathrm{nM}$ quizartinib for indicated time and MCL-1 expression was assessed in whole cell lysate by western blot.

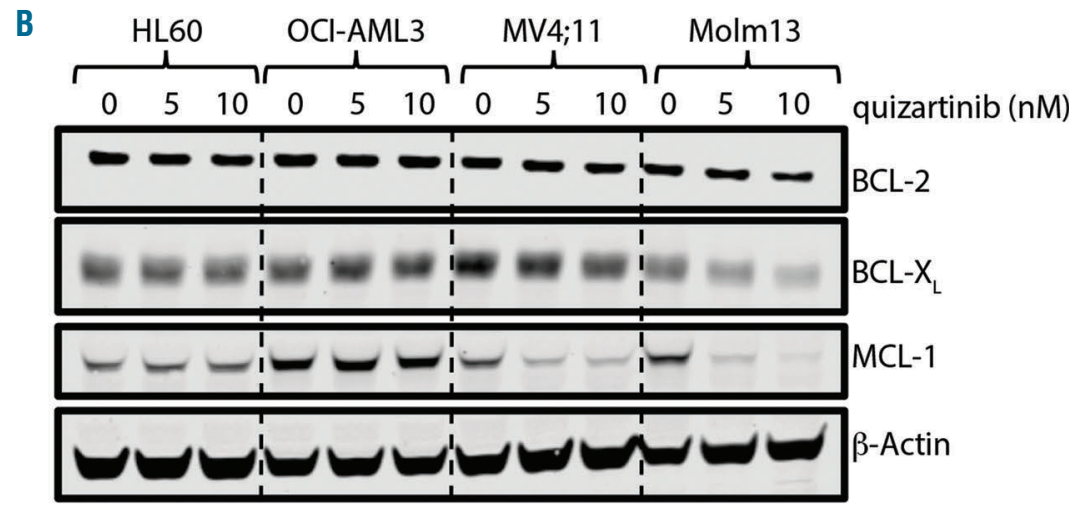

C
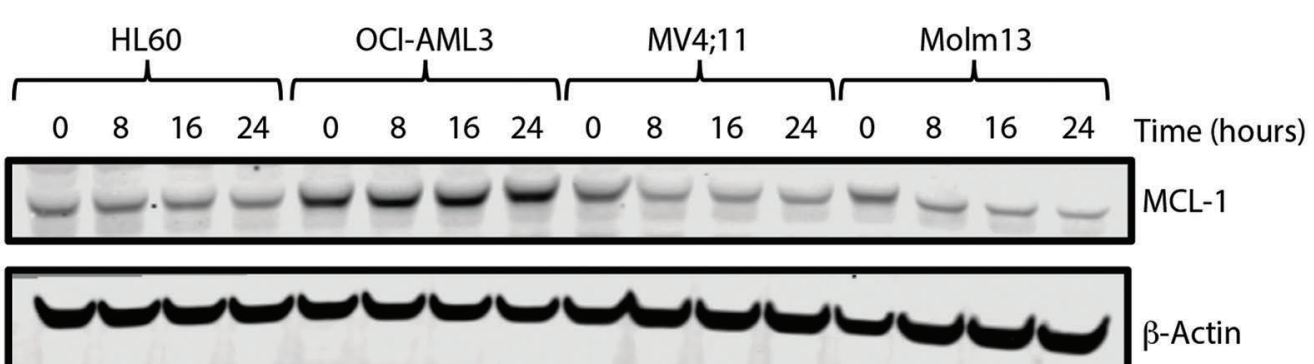

$\beta$-Actin 
While MCL-1 inhibition (AMG 176) plus quizartinib improved survival, the response was inferior to venetoclax or navitoclax (BCL-2/BCL-X ${ }_{\mathrm{L}}$ ) combined with quizartinib (Figure $4 \mathrm{~A}, \mathrm{C}$ ), demonstrating that inhibition of BCL-2 promotes enhanced anti-tumor efficacy compared to targeting MCL-1 in combination with FLT3-ITD inhibition. Co-targeting of BCL-2 and MCL-1 (venetoclax + AMG
176) resulted in shorter median survival compared with inhibition of BCL-2, BCL-X $\mathrm{L}_{\mathrm{L}}$ and MCL-1 (navitoclax + AMG 176; Figure 4B-C) indicating that in order to achieve maximum efficacy in the MV4;11 model in vivo, inhibition of all three anti-apoptotic proteins may be required. Quizartinib plus venetoclax was superior to navitoclax plus AMG 176, suggesting that FLT3-ITD inhibition

A

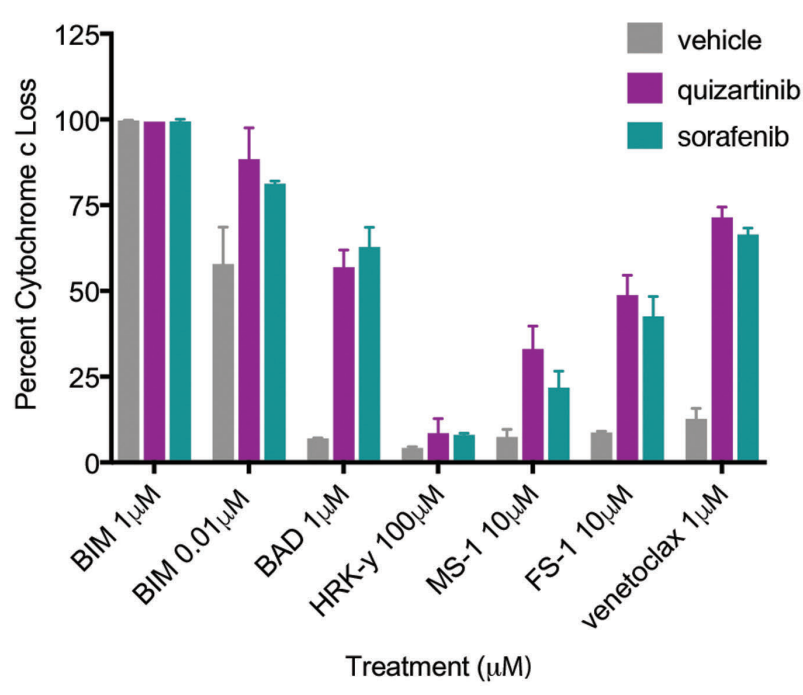
Figure 7. FLT3-ITD inhibition primed cells to BCL-2 dependence. (A) MV4;11 cells were pre-treated for 6 hours with vehicle, $5 \mathrm{nM}$ quizartinib or $20 \mathrm{nM}$ sorafenib and depletion of intracellular cytochrome $\mathrm{c}$ was deter- mined following 1 hour exposure to BIM, BAD, HRK, MS 1 and FS-1 peptides or venetoclax at the indicated con- centrations by flow cytometry. Data represents average \pm standard deviation within the experiment. (B) MV4;11 and Molm13 cells were treated with $10 \mathrm{nM}$ quizartinib for 24 hours followed by immunoprecipitation for BIM or BAK as indicated followed by Western blot analysis for BCL-2. (C) Cell lines were treated with $100 \mathrm{nM}$ veneto- clax, $10 \mathrm{nM}$ quizartinib or the combination for 24 hours and cell lysates were assessed by Western blot for BCL- 2 family proteins, cleaved caspase- 3 and cleaved PARP as indicated. Data represents two independent experi- ments. FLT3-ITD: Fms-like tyrosine kinase 3 internal tan- dem duplication.

B
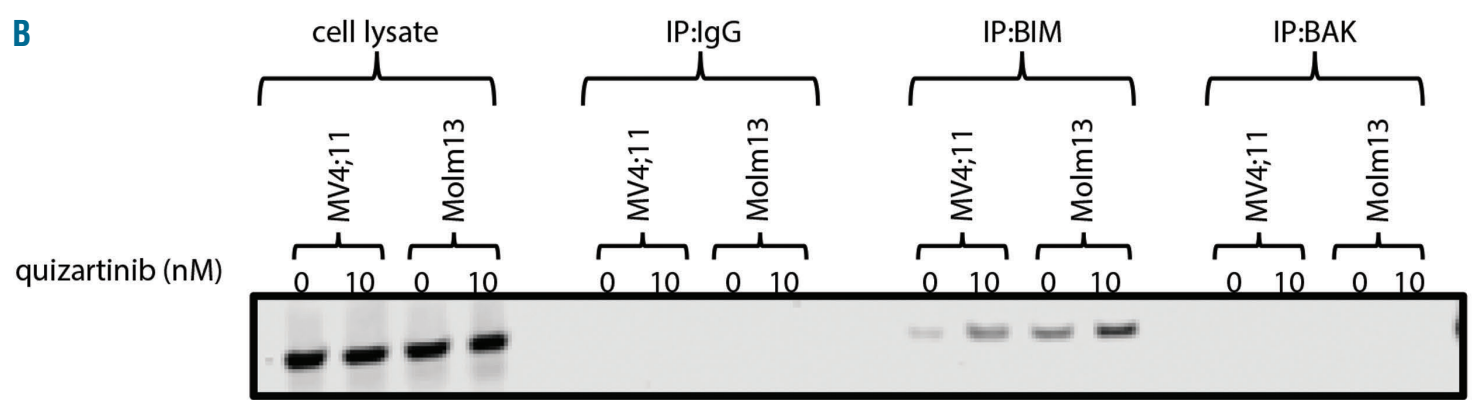

WB: BCL-2

C

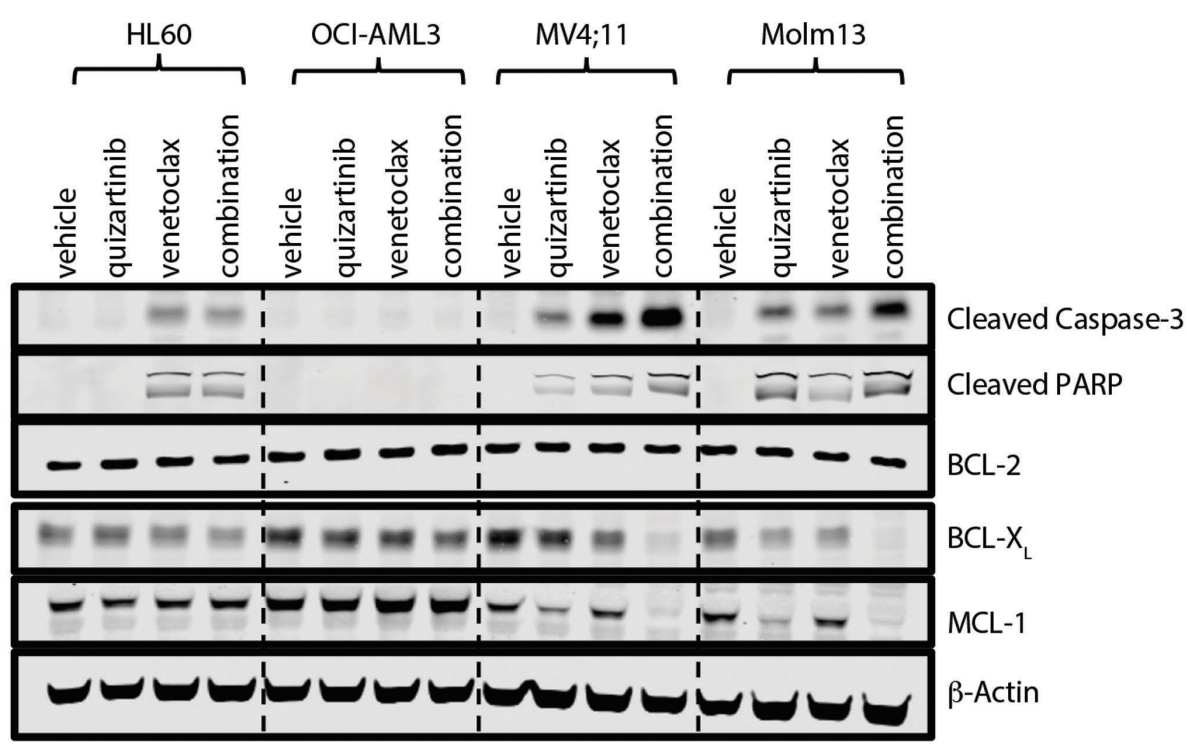


blocked additional pro-survival nodes. Treatments were well tolerated based on minimal changes in body weight (Online Supplementary Figure S4). These data underscore the need to target all three BCL-2 family members to robustly induce FLT3-ITD+ leukemia cell death in vivo.

\section{Maximum efficacy is demonstrated in vitro when simultaneously inhibiting BCL-2, BCL- $X_{L}$ and MCL-1 in FLT3-ITD+ cell lines}

In order to further dissect the combination of venetoclax and quizartinib, survival and signaling pathways were pharmacologically investigated in vitro. FLT3-ITD+ and FLT3-WT (HL60 and OCI-AML3) cell lines were treated in a dose matrix with quizartinib and venetoclax and analyzed by the Bliss independence model ${ }^{34}$ to determine synergy. Bliss scores added within a cell line are referred to as a Bliss sum and $>100$ was considered highly synergistic. Only the FLT3-ITD+ cell lines showed positive Bliss scores, indicating the combination is synergistic in vitro (Figure 5A; Online Supplementary Figure S5A). Confirming the Bliss analysis, co-treatment with low dose quizartinib and venetoclax reduced proliferation and increased apoptosis in the FLT3-ITD+ cell lines (Figure 5B; Online Supplementary Figure S5B). Venetoclax combined with sorafenib or midostaurin also reduced viability (Online Supplementary Figure S5C) while no combination effect was observed in FLT3-WT cell lines (Online Supplementary Figure $S 5 B-C$ ) indicating FLT3-ITD-specific response. In order to pharmacologically assess the contribution of MCL-1 and BCL- $\mathrm{X}_{\mathrm{L}}$ in vitro, AMG 176 and A1331852 $\left(B C L-X_{L}\right.$ specific) ${ }^{9}$ were utilized in combination with quizartinib. Bliss analysis demonstrated that quizartinib combined with AMG 176 or A1331852 was not synergistic in vitro (Figure $5 \mathrm{C}$ ).

In order to understand the dependence of FLT3-ITD+ cell lines on BCL-2, BCL- $\mathrm{X}_{\mathrm{L}}$ and $\mathrm{MCL}-1$ in vitro, combinations of venetoclax, navitoclax (BCL-2/BCL-X $\mathrm{L}$ ), A1331852 $\left(\mathrm{BCL}-\mathrm{X}_{\mathrm{L}}\right)$ and AMG $176(\mathrm{MCL}-1)$ were tested. In both cell lines, co-inhibition of BCL-2, BCL- $\mathrm{X}_{\mathrm{L}}$ and MCL-1 (AMG $176+$ navitoclax) was as synergistic as quizartinib plus venetoclax highlighting that all three proteins mediated survival. However, differential responses between cell lines were observed for other combinations. For the Molm13 cell line, venetoclax plus A1331852 (BCL-X $\mathrm{L}_{\mathrm{L}}$ ) or AMG 176 (MCL-1) showed comparable or more synergy than quizartinib plus venetoclax, respectively and AMG 176 plus A1331852 (MCL-1 and BCL-X inhibition) was least synergistic (Figure 5D; Online Supplementary Figure $S 6 A$ ). These data suggest that BCL-2 may play an important survival role in Molm 13 cells and needs to be co-targeted. The combination of navitoclax plus AMG 176 was less effective than venetoclax plus AMG 176, which could be a result of the reduced potency of navitoclax toward BCL-2 compared to venetoclax. ${ }^{9}$ This is reflected by the combination activity observed at lower doses of venetoclax ( $<4 \mathrm{nM}$ ) when combined with AMG 176 compared to navitoclax plus AMG 176 (Online Supplementary Figure S6A).

For the MV4;11 cell line, synergy scores were similar for venetoclax plus AMG 176 (MCL-1) and AMG 176 plus A1331852 (MCL-1 and BCL- $\mathrm{X}_{\mathrm{L}}$ inhibition) indicating that MCL-1 inhibition is synergistic with BCL-2 or BCL-X9 inhibition in vitro (Figure 5D; Online Supplementary Figure S6A). Interestingly, in vitro venetoclax plus AMG 176 and navitoclax plus AMG 176 responses were similar whereas in vivo navitoclax plus AMG 176 was superior to venetoclax plus AMG 176 (Figure 4B). Assessment of protein expression in cells grown in vitro versus in vivo revealed increased BCL- $\mathrm{X}_{\mathrm{L}}$ expression and decreased BCL-2 expression in vivo (Online Supplementary Figure $S 6 B$ ) which could account for the greater dependence on BCL- $\mathrm{X}_{\mathrm{L}}$ for survival in vivo. Venetoclax plus $\mathrm{A} 1331852\left(\mathrm{BCL}-\mathrm{X}_{\mathrm{L}}\right)$ was not synergistic in vitro (Figure $5 \mathrm{D})$, consistent with minimal navitoclax activity in vivo (Figure 4B). These data further demonstrate the need for simultaneous inhibition of BCL-2, BCL$\mathrm{X}_{\mathrm{L}}$ and MCL-1 in order to achieve maximal anti-tumor activity in FLT3-ITD+ models as each of these proteins appear to mediate survival.

\section{BCL-X and MCL-1 expression is regulated by FLT3-ITD in vitro}

Given that BCL- $\mathrm{X}_{\mathrm{L}}$ and MCL-1 are described to be downstream of FLT3-ITD, we evaluated the effect of FLT3 inhibition on protein expression. Quizartinib treatment resulted in kinase inhibition only in FLT3-ITD+ cell lines as confirmed by reduced phosphorylation of downstream effectors ERK, AKT and STAT5 (Figure 6A). While protein levels remained unchanged in FLT3-WT cells, quizartinib reduced $B C L-X_{L}$ protein by $20-40 \%$ and $M C L-1$ protein by $60-80 \%$, but did not alter BCL-2 expression in FLT3-ITD+ cell lines (Figure 6B; Online Supplementary Figure S7A). Sorafenib and midostaurin also reduced MCL-1 protein in the FLT3-ITD+ cell lines (Online Supplementary Figure S7B), confirming FLT3-ITD specificity and consistent with reported data. ${ }^{31}$ A shorter treatment period demonstrated maximum MCL-1 downregulation as early as 8 hours post FLT3-ITD inhibition (Figure 6C; Online Supplementary Figure S7C) reflective of the short half-life for MCL-1 ( 2 hours). ${ }^{35}$ Assessment of gene expression showed minimal decrease in mRNA expression following quizartinib treatment (Online Supplementary Figure S7D), suggesting posttranscriptional regulation. Further, proteasome (MG132) but not caspase (Z-VAD-FMK) inhibition rescued loss of MCL-1 protein induced by quizartinib (Online Supplementary Figure S7E). Given the long half-life of BCL-X $\mathrm{L}_{\mathrm{L}}\left(\sim 20\right.$ hours $\left.^{36}\right)$ and toxicity associated with MG132, $B C L-X_{L}$ protein changes were not captured. However, these data support that BCL- $\mathrm{X}_{\mathrm{L}}$ and MCL-1 are indirectly regulated post-transcriptionally downstream of FLT3-ITD.

\section{FLT3-ITD+ cells become BCL-2 dependent following quizartinib treatment}

In order to determine if FLT3-ITD inhibition altered survival dependence, dynamic $\mathrm{BH} 3$ profiling was utilized. MV4;11 cells were pre-treated with quizartinib or sorafenib followed by treatment with BH3 domain mimetic peptides and assessed for mitochondrial cytochrome c (Cytc) loss. ${ }^{37}$ FLT3-ITD inhibition primed cells toward apoptosis as determined by increased Cytc loss when co-treated with low-dose BIM peptide $(0.01 \mu \mathrm{M})$ compared to vehicle-treated (Figure 7A). FLT3ITD inhibition increased Cytc loss when co-treated with BAD, MS-1 and FS-1 peptides, suggesting dependence on BCL-2/BCL-X $\mathrm{L}, \mathrm{MCL}-1$ and BCL2-related protein A1 (A1) proteins, respectively, with $\mathrm{BAD}\left(\mathrm{BCL}-2 / \mathrm{BCL}-\mathrm{X}_{\mathrm{L}}\right)$ peptide showing greatest activity (Figure $7 \mathrm{~A})$. Co-treatment with HRK peptide (BCL- $\mathrm{X}_{\mathrm{L}}$ ) demonstrated little activity, while venetoclax combined with FLT3-ITD inhibition resulted in Cytc loss similar to BAD peptide confirming shifted survival dependency to BCL-2 in vitro (Figure 7A). Further, 
quizartinib treatment increased BIM:BCL-2 complexes in FLT3-ITD+ cell lines (Figure 7B), and co-treatment of cells with quizartinib and venetoclax caused greater reduction in BCL- $\mathrm{X}_{\mathrm{L}}$ and MCL-1 protein expression in FLT3-ITD+ cell lines (Figure 7C) compared to single agents. Therefore, FLT3-ITD inhibition switched survival dependence to BCL-2 in vitro, providing mechanistic rationale for the synergistic activity of quizartinib and venetoclax.

\section{$\mathrm{BCL}-\mathrm{X}_{\mathrm{L}}$ and MCL-1 expression is maintained in vitro by multiple FLT3-ITD downstream pathways}

In order to determine the FLT3-ITD signaling pathways regulating expression of $B C L-2, B C L-X_{L}$ and $M C L-1$, selective kinase inhibitors pictisilib (PIBK), cobimetinib (MEK) or ruxolitinib (JAK) were utilized. BCL- $\mathrm{X}_{\mathrm{L}}$ and BCL-2 protein were unchanged by any treatment in the FLT3-ITD+ cell lines while PI3K inhibition slightly reduced MCL-1 protein across all cell lines and JAK inhibition reduced MCL-1 protein only in FLT3-ITD+ cell lines (Online Supplementary Figure S8A-B). In order to pharmacologically test dependence on these pathways, Bliss analysis was performed. PI3K and BCL-2 co-inhibition was the most synergistic in the FLT3-ITD+ cell lines followed by MEK and BCL-2 co-inhibition. Although ruxolitinib reduced MCL-1 protein expression, the combination was not synergistic in FLT3-ITD+ cell lines (Online Supplementary Figure S8C). All Bliss sums ranked less than quizartinib plus venetoclax. The reduced synergy and inability of individual downstream pathways to modulate BCL-X and MCL-1 protein support directly targeting FLT3-ITD rather than downstream effectors in combination with venetoclax. Together, the data presented here demonstrated that the combination of quizartinib and venetoclax targets all three anti-apoptotic proteins through indirect regulation of BCL- $\mathrm{X}_{\mathrm{L}}$ and MCL-1 by FLT3-ITD inhibition and potent inhibition of BCL-2 by venetoclax.

\section{Discussion}

Although minimally active as a monotherapy, venetoclax combined with HMA or LDAC is efficacious in AML patients who are unfit for high intensity chemotherapy across diverse mutation subsets. ${ }^{18,19}$ Given this activity, there is clinical rationale for developing combination regimens that maximize efficacy while minimizing toxicity. With this in mind, we provide strong mechanistic rationale for a combination regimen of FLT3-ITD inhibition and venetoclax to be tested clinically.

FLT3 inhibitors became standard of care in FLT3-mutant AML with midostaurin plus chemotherapy in frontline AML and gilteritinib monotherapy in relapsed/refractory AML receiving Food and Drug Administration approval. However, duration of response is often short-lived and patients can relapse with aggressive disease. Therefore, combining FLT3 inhibitors with agents that potently induce apoptosis has the potential to achieve more durable responses for FLT3-ITD+ patients. Combination activity of midostaurin or gilteritinib and venetoclax in preclinical FLT3-ITD+ models has recently been described. ${ }^{31}$ However, the role of BCL-X $\mathrm{L}_{\mathrm{L}}$ in FLT3-ITD+ cells or the contribution of FLT3-ITD downstream pathways in mediating BCL-2 family dependence was not explored in depth. Here we have demonstrated that BCL$2, \mathrm{BCL}_{\mathrm{L}}$ and MCL-1 all promote survival of FLT3-ITD+ cells in vivo and that targeting of single pathways downstream of FLT3-ITD is insufficient to modulate BCL-X and MCL-1. We have provided a more thorough investigation of BCL-2 family dependencies in vitro and in vivo and demonstrate robust anti-leukemia activity of venetoclax combined with FLT3 inhibitors in FLT3-ITD+ preclinical AML models. The data presented here demonstrated that FLT3-ITD+ inhibition indirectly modulated BCL- $\mathrm{X}_{\mathrm{L}}$ and MCL-1 expression while venetoclax potently inhibited BCL-2 resulting in simultaneous targeting of all three anti-apoptotic proteins. Importantly, our studies utilized clinically achievable concentrations of inhibitors and demonstrated in vivo anti-tumor activity in models of established leukemia.

We identified that inhibition of individual signaling pathways downstream of FLT3-ITD was insufficient in modulating anti-apoptotic proteins, suggesting that multiple downstream pathways need to be simultaneously inhibited to induce apoptosis. Such functional co-operation has been described for BCR-ABL, where RAS, STAT5 and PIBK individually contribute to survival and provide overlapping anti-apoptotic signals. ${ }^{38}$ Potently targeting FLT3-ITD presents an opportunity to inhibit multiple downstream pathways to indirectly reduce BCL- $\mathrm{X}_{\mathrm{L}}$ and MCL-1 expression and switch survival dependence to BCL-2. This switch in survival dependence sensitizes mutant cells to venetoclax treatment as demonstrated by enhanced anti-tumor activity of quizartinib plus venetoclax compared to monotherapies. While mechanisms of resistance to the combination of FLT3-ITD inhibition and venetoclax were not explored here, resistance to FLT3 inhibitors is well-described and includes acquired inhibitor-resistant point mutations, ${ }^{39}$ suboptimal drug concentrations in the BM, bypass of FLT3-ITD signaling, ${ }^{40}$ and alteration of apoptotic proteins. ${ }^{41}$ Additionally, mutations in BCL-2 have been identified in chronic lymphocytic leukemia patients following relapse as a mechanism of venetoclax resistance. ${ }^{42,43}$ Based on the indirect regulation of BCL- $\mathrm{X}_{\mathrm{L}}$ and MCL-1 by FLT3-ITD signaling described here, potential resistance mechanisms to the combination of venetoclax and FLT3-ITD inhibition could include inhibitor-resistant point mutations that interfere with FLT3-ITD signaling inhibition or activating mutations in parallel signaling pathways, both of which would likely alter BCL-2 family expression. Investigation into resistance mechanisms is the focus of future work.

Importantly, venetoclax plus quizartinib combination activity was demonstrated in vivo in FLT3-ITD heterozygous (Molm13) and homozygous (MV4;11) preclinical models. Superior long-term survival and disease free state was observed in the MV4;11 xenograft model, which could reflect stronger FLT3-ITD addiction in the homozygous model as described by Pratz et al ${ }^{44}$ or result from different secondary mutations in the models. Combination activity was also demonstrated in a PDX model (Online Supplementary Figure S3A) with co-occurring FLT3/DNMT3A/NPM1 mutations, a subset associated with heavy disease burden and poor event free survival compared to non-triplet mutant patients. ${ }^{45,46}$ Notably, gilteritinib improved survival in FLT3/DNMT3A/NPM1 mutant patients compared to chemotherapy ${ }^{47}$ and our data indicate venetoclax may further enhance gilteritinib activity in this subset. While additional studies are required to parse out biological differences between FLT3-ITD heterozygous and homozygous mutations and the role of co- 
occurring mutations, the data presented here describes combination activity for venetoclax and FLT3 inhibition in multiple FLT3-ITD+ preclinical models.

The BM microenvironment can provide protection from cytotoxic agents and secreted factors in conditioned media from immortalized BM stromal cells increased BCL- $\mathrm{X}_{\mathrm{L}}$ (BCL2L1) mRNA expression and decreased BCL2 in primary AML samples, correlating to loss of venetoclax sensitivity compared to samples cultured in normal media. ${ }^{48}$ This altered expression was also observed in MV4;11 cells grown in vivo versus in vitro (Online Supplementary Figure $S 6 B)$. As seen in this and other studies, ${ }^{13,49}$ the Molm13 and MV4;11 cell lines are sensitive to venetoclax in vitro, however, when grown in vivo sensitivity is greatly lost (Figure 1). Therefore, the contribution of anti-apoptotic proteins in promoting survival may be underestimated outside of the tumor microenvironment. In support, quizartinib monotherapy eliminated tumor cells from the periphery $(<5 \%$ hCD $45+$ cells) but not the spleen or BM of the FLT3ITD+ PDX model while quizartinib plus venetoclax more completely eliminated leukemic cells from the tumor microenvironment (Figure 3B). Importantly, anti-tumor activity was achieved with clinically relevant doses of both venetoclax and quizartinib.

Together, the preclinical data presented here provides strong mechanistic rationale for the combination of venetoclax and FLT3 inhibitors in FLT3-ITD+ AML. Indeed, clinical investigation has been initiated through a phase $1 \mathrm{~b}$ multi-center study of venetoclax and gilteritinib in $R / R$ AML (clinicaltrials gov. Identifier: 03625505) and a phase 1b/II study of quizartinib and venetoclax in R/R FLT3mutated AML (clinicaltrials gov. Identifier: 03735875). Perl et al. recently reported that the combination of venetoclax and gilteritinib is well-tolerated with febrile neutropenia $(47 \%)$, anemia $(27 \%)$, thrombocytopenia and neutropenia (each $7 \%$ ) being the most common treat- ment-related adverse events. In the dose escalation portion of this study, the drug combination showed high response rates, with $90 \%$ of FLT3 mutant patients demonstrating blast clearance. ${ }^{50}$ The data collected from these on-going trials will be important to define the mechanistic activity and safety of venetoclax combined with FLT3ITD inhibition in patients.

\section{Disclosures}

Venetoclax is developed in collaboration between Genentech, Inc. and AbbVie. RSM, VM, EFC, MD, DS and EAL are current or former employees of Genentech, Inc.. NPS received research funding from Bristol-Myers Squibb. MK is a consultant for AbbVie, Genentech, F. Hoffman La-Roche; served as advisory board member for F. Hoffman La-Roche and AbbVie; holds shares from Reata Pharmaceuticals; honoraria from Amgen, Abbvie, Genentech; research funding from AbbVie, Genentech, Eli Lilly, Cellectis, Calithera, Stemline, Threshold, Flexus Biosciences, Novartis, Ablynx, Agios and Amgen. All other authors declare no conflicts of interest.

\section{Contributions}

$R S M, Q Z, R D, E F C, M D, N P S, M K, D S$ and EAL designed experiments and analyzed data; RSM, EAL, $Q Z, R D$, $V M K, A C, V M$ and JR performed experiments; RSM, $M D, D S$ and EAL wrote the manuscript. All authors reviewed and edited the manuscript.

\section{Acknowledgments}

The authors acknowledge Kyle Edgar for support on the Bliss analysis, and Aaron Logan and colleagues at the UCSF tissue bank for providing primary patient samples (supported by the UCSF Cancer Center Support Grant). The authors also acknowledge and thank the Genentech dosing core, cell line core group, animal resources personnel and veterinary staff for their assistance and contributions to this project.

\section{References}

1. Levis M, Small D. FLT3: ITDoes matter in leukemia. Leukemia. 2003;17(9):1738-1752

2. Yamamoto Y, Kiyoi H, Nakano Y, et al. Activation mutation of D835 within the activation loop of FLT3 in human hematologic malignancies. Blood. 2001;97(8):24342439

3. Choudhary C, Muller-Tidow C, Berdel WE, Serve H. Signal transduction of oncogenic Flt3. Int J Hematol. 2005-82(2):93-99.

4. Hayakawa F, Towatari M, Kiyoi $\mathrm{H}$, et al. Tandem-duplicated Flt3 constitutively activates STAT5 and MAP kinase and introduces autonomous cell growth in IL3dependent cell lines. Oncogene. 2000; 19:624-631.

5. Mizuki M, Fenski R, Halfter $\mathrm{H}$, et al. Flt3 mutations from patients with acute myeloid leukemia induce transformation of $32 \mathrm{D}$ cells mediated by the Ras and STAT5 pathways. Blood. 2000:96(12):3907-3914.

6. Czabotar PE, Lessene G, Strasser A, Adams JM. Control of apoptosis by the BCL-2 protein family: implications for physiology and therapy. Nat Rev Mol Cell Biol. 2014;15(1):49-63.

7. Souers AJ, Leverson JD, Boghaert ER, et al. ABT-199, a potent and selective BCL-2 inhibitor, achieves antitumor activity while sparing platelets. Nat Med. 2013;19(2):202-
208.

8. Tse C, Shoemaker AR, Adickes J, et al. ABT263: a potent and orally bioavailable $\mathrm{Bcl}-2$ family inhibitor. Cancer Res. 2008;68(9):3421-3428.

9. Leverson JD, Phillips DC, Mitten MJ, et al. Exploiting selective BCL-2 family inhibitors to dissect cell survival dependencies and define improved strategies for cancer therapy. Sci Transl Med. 2015;7(279):279ra240.

10. Kotschy A, Szlavik Z, Murray J, et al. The MCL1 inhibitor S63845 is tolerable and effective in diverse cancer models. Nature. 2016;538(7626):477-482.

11. Caenepeel S, Brown SP, Belmontes B, et al. AMG 176, a selective MCL1 inhibitor, is effective in hematologic cancer models alone and in combination with established therapies. Cancer Discov. 2018;8(12):1582 1597

12. Bose P, Gandhi V, Konopleva M. Pathways and mechanisms of venetoclax resistance. Leuk Lymphoma. 2017;58(9):1-17

13. Pan R, Hogdal LJ, Benito JM, et al. Selective BCL-2 inhibition by ABT-199 causes on-target cell death in acute myeloid leukemia. Cancer Discov. 2014;4(3):362-375.

14. Konopleva M, Pollyea DA, Potluri J, et al. Efficacy and biological correlates of response in a Phase II Study of Venetoclax monotherapy in Patients with acute myelogenous leukemia. Cancer Discov. 2016;6(10):11061117
15. Punnoose EA, Leverson JD, Peale F, et al. Expression profile of BCL-2, BCL-XL, and MCL-1 predicts pharmacological response to the BCL-2 selective antagonist venetoclax in multiple myeloma models. Mol Cancer Ther. 2016;15(5):1132-1144.

16. Kumar S, Kaufman JL, Gasparetto C, et al. Efficacy of venetoclax as targeted therapy for relapsed/refractory $\mathrm{t}(11 ; 14)$ multiple myeloma. Blood. 2017;130(22):2401-2409.

17. Stilgenbauer S, Eichhorst B, Schetelig J, et al Venetoclax for patients with chronic lymphocytic leukemia With 17 p deletion: results from the full population of a Phase II pivotal Trial. J Clin Oncol. 2018;36(19):1973-1980.

18. Pollyea DA, Pratz KW, Jonas BA, et al Venetoclax in combination with hypomethylatling agents induces rapid, deep, and durable responses in patients with AML ineligible for intensive therapy. Blood. 2018;132(Suppl_1):285.

19. Wei AH, Montesinos P, Ivanov V, MD, et al Venetoclax plus LDAC for patients with untreated AML ineligible for intensive chemotherapy: phase 3 randomized placebo-controlled trial. Blood. 2020; 135(24) 2137-2145.

20. Chyla B, Daver N, Doyle K, et al. Genetic biomarkers of sensitivity and resistance to venetoclax monotherapy in patients With relapsed acute myeloid leukemia. Am J Hematol. 2018;93(6):E202-E205.

21. DiNardo CD, Tiong IS, Quaglieri A, et al. 
Molecular patterns of response and treatment failure after frontline venetoclax combinations in older patients with AML. Blood. 2020;135(11):791-803.

22. Fulda S. Modulation of mitochondrial apoptosis by PI3K inhibitors. Mitochondrion. 2013;13(3):195-198.

23. Thomas LW, Lam C, Edwards SW. Mcl-1; the molecular regulation of protein function. FEBS Lett. 2010;584(14):2981-2989.

24. Grad JM, Zeng X-R, Boise LH. Regulation of Bcl-XL: a little bit of this and a little bit of STAT. Curr Opin Oncol. 2000;12(6):543-549.

25. Alvarez J, Frank D. Genome-wide analysis of STAT target genes: elucidating the mechanism of STAT-mediated oncogenesis. Cancer Biol Ther. 2004;3(11):1045-1050.

26. Yoshimoto G, Miyamoto T, JabbarzadehTabrizi S, et al. FLT3-ITD up-regulates MCL1 to promote survival of stem cells in acute myeloid leukemia via FLT3-ITD-specific STAT5 activation. Blood. 2009; 114(24):5034-5043.

27. Minami Y, Yamamoto K, Kiyoi H, Ueda R, Saito $H$, Naoe T. Different antiapoptotic pathways between wild-type and mutated FLT3: insights into therapeutic targets in leukemia. Blood. 2003;102(8):2969-2975.

28. Spiekermann K, Dirschinger RJ, Schwab R, et al. The protein tyrosine kinase inhibitor SU5614 inhibits FLT3 and induces growth arrest and apoptosis in AML-derived cell lines expressing a constitutively activated FLT3. Blood. 2003;101(4):1494-1504.

29. Kasper S, Breitenbuecher F, Heidel F, et al. Targeting MCL-1 sensitizes FLT3-ITD-positive leukemias to cytotoxic therapies. Blood Cancer J. 2012;2(3):e60.

30. Bagrintseva K, Geisenhof S, Kern R, et al. FLT3-ITD-TKD dual mutants associated with AML confer resistance to FLT3 PTK inhibitors and cytotoxic agents by overexpression of $\mathrm{Bcl}-\mathrm{x}(\mathrm{L})$. Blood. 2005; 105(9):3679-3685.

31. Ma J, Zhao S, Qiao X, et al. Inhibition of Bcl2 synergistically enhances the antileukemic activity of midostaurin and gilteritinib in preclinical models of FLT3-mutated acute myeloid leukemia. Clin Cancer Res. 2019; 25(22):6815-6826

32. Zarrinkar PP, Gunawardane RN, Crame $\mathrm{MD}$, et al. AC220 is a uniquely potent and selective inhibitor of FLT3 for the treatment of acute myeloid leukemia (AML). Blood. 2009;114(14):2984-2992.

33. Levis MJ, Cortes JE, Gammon GM, Trone D, Kang D, Li J. Laboratory and clinical investigations to identify optimal dosing strategy for quizartinib (AC220) monotherapy in FLT3-ITD positive relapsed/refractory acute myeloid leukemia. Blood. 2016; 128(22) 4042.

34. Foucquier J, Guedj M. Analysis of drug combinations: current methodological landscape. Pharmacol Res Perspect. 2015; 3(3):e00149.

35. Yang T, Kozopas KM, Craig RW. The intracellular distribution and pattern of Mcl-1 overlap with, but are not identical to, those of Bcl-2. J Cell Biol. 1995;128(6):1173-1184.

36. Rooswinkel RW, van de Kooij B, de Vries E, et al. Antiapoptotic potency of Bcl-2 proteins primarily relies on their stability, not binding selectivity. Blood. 2014; 123(18): 2806-2815.

37. Del Gaizo Moore V, Letai A. BH3 profiling-measuring integrated function of the mitochondrial apoptotic pathway to predict cell fate decisions. Cancer Lett. 2013;332(2):202205.

38. Sonoyama J, Matsumura I, Ezoe S, et al. Functional cooperation among Ras, STAT5, and phosphatidylinositol 3-kinase is required for full oncogenic activities of BCR/ABL in K562 cells. J Biol Chem. 2002; 277(10):8076-8082

39. Smith CC, Wang Q, Chin CS, et al. Validation of ITD mutations in FLT3 as a therapeutic target in human acute myeloid leukaemia. Nature. 2012;485(7397):260-263.

40. McMahon CM, Ferng T, Canaani J, et al Clonal selection with RAS pathway activation mediates secondary clinical resistance to selective FLT3 inhibition in acute myeloid leukemia. Cancer Discov. 2019;9(8):1050 1063 .
41. Naqvi K, Konopleva M, Ravandi F. Targeted therapies in acute myeloid leukemia: a focus on FLT-3 inhibitors and ABT199. Expert Rev Hematol. 2017;10(10):863-874.

42. Blombery P, Anderson MA, Gong J-n, et al Acquisition of the recurrent Gly101Va mutation in BCL2 confers resistance to venetoclax in patients with progressive chronic lymphocytic leukemia. Cancer Discov. 2019;9(3):342-353.

43. Tausch E, Close W, Dolnik A, et al. Venetoclax resistance and acquired BCL2 mutations in chronic lymphocytic leukemia. Haematologica. 2019;104(9):434-437.

44. Pratz KW, Sato T, Murphy KM, Stine A Rajkhowa T, Levis M. FLT3-mutant allelic burden and clinical status are predictive of response to FLT3 inhibitors in AML. Blood. 2010;115(7):1425-1432

45. Loghavi S, Zuo Z, Ravandi F, et al. Clinica features of de novo acute myeloid leukemia with concurrent DNMT3A, FLT3 and NPM1 mutations. J Hematol Oncol. 2014;7:74.

46. Bezerra MF, Lima AS, Piqué-Borràs M-R, et al. Co-occurrence of DNMT3A, NPM1, FLT3 mutations identifies a subset of acute myeloid leukemia with adverse prognosis. Blood. 2020;135(11):870-875.

47. Perl AE, Martinelli G, Cortes JE, et al. Gilteritinib or chemotherapy for relapsed or refractory FLT3-mutated AML. N Engl J Med. 2019;381(18):1728-1740

48. Karjalainen R, Pemovska T, Popa M, et al JAK1/2 and BCL2 inhibitors synergize to counteract bone marrow stromal cellinduced protection of AML. Blood. 2017; 130(6):789-802

49. Niu X, Wang G, Wang Y, et al. Acute myeloid leukemia cells harboring MLL fusion genes or with the acute promyelocytic leukemia phenotype are sensitive to the Bcl-2-selective inhibitor ABT-199. Leukemia. 2014;28(7):1557-1560.

50. Perl AE, Daver NG, Pratz KW, et al. Venetoclax in combination with gilteritinib in patients with relapsed/refractory acute myeloid leukemia: a Phase $1 \mathrm{~b}$ study. Blood. 2019;134(Supp_1):3910. 